Mathematical Modelling and Analysis

Volume 11 Number 2, 2006, PAGes 161-172

(c) 2006 Technika ISSN 1392-6292 print, ISSN 1648-3510 online

\title{
NUMERICAL ANALYSIS OF THE WAVE PROPAGATION IN A COMPOSITE MEDIUM
}

\author{
P. KATAUSKIS and V. SKAKAUSKAS \\ Vilnius University \\ Naugarduko str. 24, 03225 Vilnius, Lithuania \\ Received October 10, 2005; revised March 17, 2006; published online May 25, 2006 \\ E-mail: pranas. katauskis@mif.vu. 1t; vladas.skakauskas@mat.vu. It
}

\begin{abstract}
We consider the wave propagation process in a 2-dimensional material structure composed of random oriented orthotropic crystals and analyse numerical results for the diffraction problem and the problem of waves interaction with a free boundary.

Key words: orthotropic crystals, wave propagation, diffraction, numerical algorithms
\end{abstract}

\section{Introduction}

Modelling of short wave propagation process is of key importance for solution of very different problems [2]. We mention only identification and recognition of defects in solids [1,3]. In paper [2] a model of a linearly elastic composite medium consisting of a matrix containing a set of orthotropic crystals with randomly oriented anisotropy axes was proposed and some numerical results for the wave interaction with a free boundary were given.

In the present paper we use the model given in [2] and examine the waves diffraction and waves interaction with a free boundary. Since equations of the model are of the hyperbolic type there may occur waves caused by the discretization in the computer modelling. As a test problem we examine the one-dimensional model. We give the explicit formula for the solution of this problem and solve it numerically.

The paper is organized as follows. In Section 2 the one-dimensional case is considered. In Section 3 the formulation of the problems in two-dimensional case is given. Numerical results are presented in Section 4 . Section 5 concludes the paper. 


\section{The One-Dimensional Problem}

The interaction of waves with a free boundary in one-dimensional case can be described by the model

$$
\left\{\begin{array}{l}
\frac{\partial^{2} u}{\partial t^{2}}=b^{2} \frac{\partial^{2} u}{\partial x^{2}}, \quad x \in(0,1), t>0, \\
\left.\frac{\partial u}{\partial x}\right|_{x=0}=\left\{\begin{array}{cc}
-\mu(t), & t \leq T, \\
0, & t>T, \\
\left.\frac{\partial u}{\partial x}\right|_{x=1}=0, & \\
\left.u\right|_{t=0}=\left.\frac{\partial u}{\partial t}\right|_{t=0}=0 .
\end{array}\right.
\end{array}\right.
$$

Here we have the following coefficients

$$
T \leq \frac{1}{b}, \quad \mu \in C^{1}[0, T], \quad \mu(0)=\mu^{\prime}(0)=\mu(T)=\mu^{\prime}(T)=0, \quad \int_{0}^{T} \mu(\tau) d \tau=0,
$$

where the prime indicates differentiation. Define $f(t)=b \int_{0}^{t} \mu(\tau) d \tau$. The solution of problem (2.1) is expressed by the formulas (see Fig. 1):

$$
\begin{aligned}
& u(t, x)=0 \quad \text { in } D_{0}=\left\{0 \leq t \leq \frac{x}{b}\right\}, \\
& u(t, x)=f\left(t-\frac{x}{b}\right) \quad \text { in } D_{1}=\left\{\frac{x}{b} \leq t \leq \min \left(T+\frac{x}{b}, \frac{2-x}{b}\right)\right\}, \\
& u(t, x)=f\left(t-\frac{x}{b}\right)+f\left(t+\frac{x-2}{b}\right) \quad \text { in } D_{2}=\left\{\frac{2-x}{b} \leq t \leq T+\frac{x}{b}\right\}, \\
& u(t, x)=0 \quad \text { in } D_{3}=\left\{T+\frac{x}{b} \leq t \leq \frac{2-x}{b}\right\}, u(t, x)=f\left(t+\frac{x-2}{b}\right) \text { in } D_{4}, \\
& D_{4}=\left\{\max \left(\frac{2-x}{b}, T+\frac{x}{b}\right) \leq t \leq \min \left(T+\frac{2-x}{b}, \frac{2+x}{b}\right)\right\}, \\
& u(t, x)=f\left(t+\frac{x-2}{b}\right)+f\left(t-\frac{2+x}{b}\right) \text { in } D_{5}=\left\{\frac{2+x}{b} \leq t \leq T+\frac{2-x}{b}\right\},
\end{aligned}
$$

and so on. 
Numerical Analysis of the Wave Propagation in a Composite Medium 163

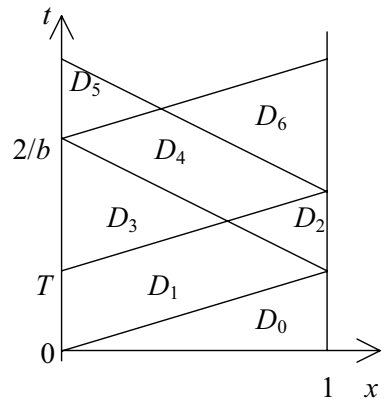

Figure 1. Sketch of the 1D domain for problem (2.1).

The diffraction problem is described by the model

$$
\left\{\begin{array}{l}
\frac{\partial^{2} u}{\partial t^{2}}=b^{2} \frac{\partial^{2} u}{\partial x^{2}}, \quad x \in(0,1) \cup(1, d), t>0 \\
\left.\frac{\partial u}{\partial x}\right|_{x=0}=\left\{\begin{array}{l}
-\mu(t), \quad t \leq T \\
0, \quad t>T
\end{array}\right. \\
\left.\frac{\partial u}{\partial x}\right|_{x=d}=0 \\
{\left[\left.u\right|_{x=1}\right]=\left[\left.b^{2} \frac{\partial u}{\partial x}\right|_{x=1}\right]=0} \\
\left.u\right|_{t=0}=\left.\frac{\partial u}{\partial t}\right|_{t=0}=0 .
\end{array}\right.
$$

Here the constant $b=b_{1}$ if $x \in[0,1), b=b_{2}$ if $x \in(1, d], \mu$ is the same as in model (2.1) and $\left[\left.u\right|_{x=1}\right]$ denotes a jump of $u$ at the point of discontinuity $x=1$. The solution of problem (2.2) can be expressed by formulas (see Fig. 2):

$$
\begin{aligned}
& u(t, x)=0 \quad \text { in } D_{0}=\left\{t \leq \frac{x}{b_{1}} \text { for } x \leq 1 \text { and } t \leq \frac{1}{b_{1}}+\frac{x-1}{b_{2}} \text { for } x \geq 1\right\}, \\
& u(t, x)=f\left(t-\frac{x}{b_{1}}\right) \quad \text { in } D_{1}=\left\{\frac{x}{b_{1}} \leq t \leq \min \left(\frac{2-x}{b_{1}}, T+\frac{x}{b_{1}}\right), x \leq 1\right\}, \\
& u(t, x)=f\left(t-\frac{x}{b_{1}}\right)+\frac{b_{1}-b_{2}}{b_{1}+b_{2}} f\left(t+\frac{x-2}{b_{1}}\right) \quad \text { in } D_{2}, \\
& D_{2}=\left\{\left(\frac{2-x}{b_{1}} \leq t \leq T+\frac{x}{b_{1}}\right), x \leq 1\right\}, \\
& u(t, x)=\frac{2 b}{b_{1}+b_{2}} f\left(t+\frac{1-x}{b_{2}}-\frac{1}{b_{1}}\right) \quad \text { in } D_{3},
\end{aligned}
$$




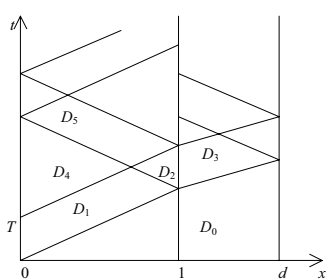

Figure 2. Sketch of the 1D domain for diffraction problem (2.2).

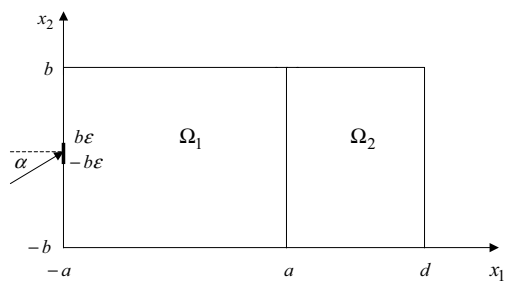

Figure 3. Sketch for the 2D domain of problem (3.1).

$$
\begin{aligned}
& D_{3}=\left\{\frac{1}{b_{1}}+\frac{x-1}{b_{2}} \leq t \leq \min \left(T+\frac{1}{b_{1}}+\frac{x-1}{b_{2}}, \frac{1}{b_{1}}+\frac{2 d-1-x}{b_{2}}\right), x \geq 1\right\}, \\
& u(t, x)=0, \quad \text { in } D_{4}=\left\{T+\frac{x}{b_{1}} \leq t \leq \frac{2-x}{b_{1}}, x \leq 1\right\} \\
& u(t, x)=\frac{b_{1}-b_{2}}{b_{1}+b_{2}} f\left(t+\frac{x-2}{b_{1}}\right), \quad \text { in } D_{5}, \\
& D_{5}=\left\{\max \left(T+\frac{x}{b_{1}}, \frac{2-x}{b_{1}}\right), \min \left(\frac{2+x}{b_{1}}, T+\frac{2-x}{b_{1}}\right), x \leq 1\right\},
\end{aligned}
$$

and so on. In what follows we use the function $\mu(t)=-q \sin ^{3} \omega t, q=$ const, $T=2 \pi / \omega$.

\section{The 2-Dimensional Problem}

Let us first examine the waves diffraction problem i.e. when waves propagate in the rectangular composite plate $\Omega=\Omega_{1}+\Omega_{2}$ (see Fig. 3) with piece-wise constant elasticity characteristics. Waves are generated by the given normal and tangent strains on a small part $(-b \varepsilon, b \varepsilon)$ of one side. The other sides are assumed to be free and the displacement vector $u$ and strains $P_{11}$ and $P_{12}$ have to be continuous at the discontinuity line $x_{1}=a$. The model consists of the equations

$$
\left\{\begin{array}{l}
\rho \frac{\partial^{2} u_{1}}{\partial t^{2}}=A_{1111} \frac{\partial^{2} u_{1}}{\partial x_{1}^{2}}+A_{1212} \frac{\partial^{2} u_{1}}{\partial x_{2}^{2}}+\left(A_{2211}+A_{1212}\right) \frac{\partial^{2} u_{2}}{\partial x_{1} \partial x_{2}} \\
\rho \frac{\partial^{2} u_{2}}{\partial t^{2}}=\left(A_{2211}+A_{1212}\right) \frac{\partial^{2} u_{1}}{\partial x_{1} \partial x_{2}}+A_{1212} \frac{\partial^{2} u_{2}}{\partial x_{1}^{2}}+A_{2222} \frac{\partial^{2} u_{2}}{\partial x_{2}^{2}}
\end{array}\right.
$$

$t>0,\left(x_{1}, x_{2}\right) \in \Omega$ subject to the conditions 
Numerical Analysis of the Wave Propagation in a Composite Medium 165

$$
\begin{aligned}
& A_{1111} \frac{\partial u_{1}}{\partial x_{1}}+A_{2211} \frac{\partial u_{2}}{\partial x_{2}}=\left\{\begin{array}{c}
-B\left(t, x_{2}\right) \cos \alpha, x_{1}=-a, x_{2}=[-b \varepsilon, b \varepsilon], t \leq 1, \\
0, \quad x_{1}=-a, x_{2} \in[-b \varepsilon, b \varepsilon], t>1, \\
0, \quad x_{1}=-a, x_{2} \in[-b, b] \backslash[-b \varepsilon, b \varepsilon], t>0, \\
0, \quad x_{1}=d, x_{2} \in[-b, b], t>0,
\end{array}\right. \\
& A_{1212}\left(\frac{\partial u_{1}}{\partial x_{2}}+\frac{\partial u_{2}}{\partial x_{1}}\right)=\left\{\begin{array}{c}
-B\left(t, x_{2}\right) \sin \alpha, x_{1}=-a, x_{2} \in[-b \varepsilon, b \varepsilon], t \leq 1, \\
0, x_{1}=-a, x_{2} \in[-b \varepsilon, b \varepsilon], t>1,
\end{array}\right. \\
& \frac{\partial u_{1}}{\partial x_{2}}+\frac{\partial u_{2}}{\partial x_{1}}=0, \quad x \in \partial \Omega \backslash\left\{x_{1}=-a, x_{2} \in[-b \varepsilon, b \varepsilon]\right\}, \\
& A_{2211} \frac{\partial u_{1}}{\partial x_{1}}+A_{2222} \frac{\partial u_{2}}{\partial x_{2}}=0, \quad x_{2}= \pm b, x_{1} \in(-a, d), \\
& \left.u_{1}\right|_{t=0}=\left.u_{2}\right|_{t=0}=0, \quad\left(x_{1}, x_{2}\right) \in \Omega, \\
& \left.\frac{\partial u_{1}}{\partial t}\right|_{t=0}=\left.\frac{\partial u_{2}}{\partial t}\right|_{t=0}=0, \quad\left(x_{1}, x_{2}\right) \in \Omega, \\
& {\left[u_{1}\right]=\left[u_{2}\right]=\left[A_{1111} \frac{\partial u_{1}}{\partial x_{1}}+A_{2211} \frac{\partial u_{2}}{\partial x_{2}}\right]=\left[A_{1212}\left(\frac{\partial u_{1}}{\partial x_{2}}+\frac{\partial u_{2}}{\partial x_{1}}\right)\right]=0,} \\
& x_{1}=a, x_{2} \in(-b, b) .
\end{aligned}
$$

Here $\rho$ is the material density,

$$
\begin{aligned}
& A_{1111}=0.983 C_{1111}+2.91 \cdot 10^{-4} C_{2222}+9.636 \cdot 10^{-3}\left(2 C_{1122}+4 C_{1212}\right), \\
& A_{2222}=2.91 \cdot 10^{-4} C_{1111}+0.983 C_{2222}+9.636 \cdot 10^{-3}\left(2 C_{1122}+4 C_{1212}\right), \\
& A_{2211}=9.636 \cdot 10^{-3}\left(C_{1111}+C_{2222}-4 C_{1212}\right)+0.983 C_{1122}, \\
& A_{1212}=9.636 \cdot 10^{-3}\left(C_{1111}+C_{2222}-2 C_{1122}\right)+0.964 C_{1212}, \\
& C_{1111}=\frac{E_{1}}{1-\nu_{21}^{2} E_{1} / E_{2}}, \quad C_{2211}=\frac{E_{1} \nu_{21}}{1-\nu_{21}^{2} E_{1} / E_{2}}, \\
& C_{2222}=\frac{E_{2}}{1-\nu_{21}^{2} E_{1} / E_{2}}, \quad C_{1212}=G_{12},
\end{aligned}
$$

where $G_{12}$ signifies the shear modulus, $E_{1}$ and $E_{2}$ mean elasticity moduli, and $\nu_{21}$ is the Poisson coefficient. Coefficients $\rho, G_{12}, E_{1}, E_{2}$, and $\nu_{21}$ are different in $\Omega_{1}$ and $\Omega_{2}$. In what follows we use the function

$$
B\left(t, x_{2}\right)=\eta \sin ^{3} \omega t\left((b \varepsilon)^{2}-x_{2}^{2}\right)^{2}, \quad \eta=\text { const }>0 .
$$

Formulas (3.3) are obtained from the equations [2] 


$$
\begin{aligned}
& A_{q q n m}=\int_{-\Theta}^{\Theta} f(\theta) \alpha_{i n} \alpha_{j m} \alpha_{s q} \alpha_{k q} d \theta C_{i j k s}, \quad q=1,2, \\
& 2 A_{12 n m}=\int_{-\Theta}^{\Theta} f(\theta) \alpha_{i n} \alpha_{j m}\left(\alpha_{s 1} \alpha_{k 2}+\alpha_{s 2} \alpha_{k 1}\right) d \theta C_{i j k s}, \\
& \alpha_{11}=\alpha_{22}=\cos \theta, \quad \alpha_{12}=-\alpha_{21}=\sin \theta, \\
& \alpha_{13}=\alpha_{23}=\alpha_{31}=\alpha_{32}=0, \quad \alpha_{33}=1, \quad \theta=\frac{\pi}{4} \\
& f(\theta)=e^{-\theta^{2} / 2 \sigma^{2}}\left(\begin{array}{ll}
2 & \int_{0}^{-x^{2} / 2 \sigma^{2}} \\
d x
\end{array}\right)^{-1} .
\end{aligned}
$$

By using formulas

$$
\begin{aligned}
& t=T \tilde{t}, \quad x=L \tilde{x}, \quad u_{k}=L \tilde{u}_{k}, \quad T=\frac{2 \pi}{\omega} \\
& E_{k}=\tilde{E}_{k} \cdot 10^{11} \frac{\mathrm{g}}{\mathrm{s}^{2} \mathrm{~mm}}, \quad G_{12}=\tilde{G}_{12} \cdot 10^{11} \frac{\mathrm{g}}{\mathrm{s}^{2} \mathrm{~mm}}
\end{aligned}
$$

we get the dimensionless form of Eqs. (3.1)

$$
\left\{\begin{array}{l}
\frac{\partial^{2} u_{1}}{\partial t^{2}}=\xi\left(A_{1111} \frac{\partial^{2} u_{1}}{\partial x_{1}^{2}}+A_{1212} \frac{\partial^{2} u_{1}}{\partial x_{2}^{2}}+\left(A_{2211}+A_{1212}\right) \frac{\partial^{2} u_{2}}{\partial x_{1} \partial x_{2}}\right) \\
\frac{\partial^{2} u_{2}}{\partial t^{2}}=\xi\left(\left(A_{2211}+A_{1212}\right) \frac{\partial u_{1}}{\partial x_{1} \partial x_{2}}+A_{1212} \frac{\partial^{2} u_{2}}{\partial x_{1}^{2}}+A_{2222} \frac{\partial^{2} u_{2}}{\partial x_{2}^{2}}\right)
\end{array}\right.
$$

with $\xi=\frac{T^{2}}{\rho L^{2}} \cdot 10^{14}$. Here the constants $A_{1111}, A_{2222}, A_{1212}$, and $A_{2211}$ are expressed by formulas $(3.3)$ with $E_{1}, E_{2}$, and $G_{12}$ replaced by $\tilde{E}_{1}, \tilde{E}_{2}$, and $\tilde{G}_{12}$, respectively. For simplicity the tilde is omitted in Eqs. (3.4). The formulation of the waves interaction with a free boundary follows from (3.1)-(3.4) by setting $d=a$.

\section{Numerical Results}

We first solve the one-dimensional model. For solving of the problem (2.2) the explicit difference scheme was applied (see [4], pp. 332-355 and p. 161) and the following constants were used:

$a=1.5, \quad q=20, \quad \omega=300, \quad T=2 \pi / \omega, \quad b_{1}=20, \quad b_{2}=5 \quad$ and $\quad b_{2}=30$.

Numerical results are exhibited in Figs. 4 and 5. In Fig. $4\left(b_{1}=20, b_{2}=5\right)$ waves 1 and 4 exhibit the original waves, waves 2,3 and 5 are reflected waves and function $u(t, x)$ is nonnegative. In Fig. $5\left(b_{1}=20, b_{2}=30\right)$ waves 1 and 


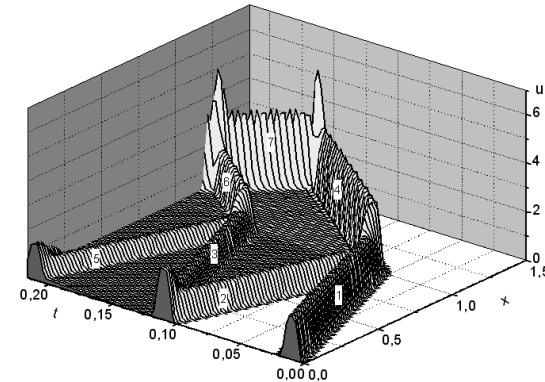

Figure 4. The graph of $u$ for problem (2.2) with $b_{1}=20, b_{2}=5$.

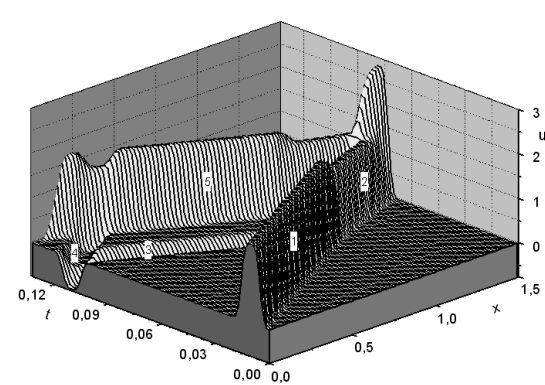

Figure 5. The graph of $u$ for problem (2.2) with $b_{1}=20, b_{2}=30$.

2 are original waves while 3,4 and 5 are reflected waves. For waves 3 and 4 function $u(x, t)$ is nonpositive.

To get the numerical solution to problem (3.4), (3.2) we use the finitedifference scheme [4]. Set

$$
\begin{aligned}
& t_{k}=k \tau, \quad 0 \leq k \leq M, \quad \tau=T / M, \\
& x_{1 i}=-a+i h_{1}, \quad 0 \leq i \leq N_{1}, \quad h_{1}=(d+a) / N_{1}, \quad N_{1}^{t}=2 a / h_{1}, \\
& x_{2 j}=-b+j h_{2}, \quad 0 \leq j \leq N_{2}, \quad h_{2}=2 b / N_{2} .
\end{aligned}
$$

Assume that $\bar{u}_{i j}^{k}=u_{1}\left(t_{k}, x_{1 i}, x_{2 j}\right)$ and $\overline{\bar{u}}_{i j}^{k}=u_{2}\left(t_{k}, x_{1 i}, x_{2 j}\right)$ and let

$$
A_{1}=\xi A_{1111}, A_{2}=\xi A_{1212}, \quad A_{3}=\xi\left(A_{2211}+A_{1212}\right), \quad A_{4}=\xi A_{2222} .
$$

The explicit difference scheme is used to approximate the differential problem

$$
\begin{aligned}
\bar{u}_{i j}^{k+1} & =b_{11}\left(\bar{u}_{i+1, j}^{k}-\bar{u}_{i j}^{k}\right)-b_{12}\left(\bar{u}_{i, j}^{k}-\bar{u}_{i-1, j}^{k}\right)+b_{13}\left(\bar{u}_{i, j-1}^{k}-2 \bar{u}_{i j}^{k}+\bar{u}_{i-1, j}^{k}\right) \\
& +b_{14}\left(\overline{\bar{u}}_{i+1, j+1}^{k}-\overline{\bar{u}}_{i+1, j-1}^{k}\right)-b_{15}\left(\overline{\bar{u}}_{i-1, j+1}^{k}-\overline{\bar{u}}_{i-1, j-1}^{k}\right)+2 \bar{u}_{i j}^{k}-\bar{u}_{i j}^{k-1} \\
\overline{\bar{u}}_{i j}^{k+1} & =b_{14}\left(\bar{u}_{i+1, j+1}^{k}-\bar{u}_{i+1, j-1}^{k}\right)-b_{15}\left(\bar{u}_{i-1, j+1}^{k}-\bar{u}_{i-1, j-1}^{k}\right)+b_{21}\left(\overline{\bar{u}}_{i+1, j}^{k}-\overline{\bar{u}}_{i, j}^{k}\right) \\
& -b_{22}\left(\overline{\bar{u}}_{i+1, j}^{k}-\overline{\bar{u}}_{i+1, j}\right)+b_{23}\left(\overline{\bar{u}}_{i, j-1}^{k}-2 \overline{\bar{u}}_{i, j}^{k}+\overline{\bar{u}}_{i, j+1}^{k}\right)+2 \overline{\bar{u}}_{i, j}^{k}-\overline{\bar{u}}_{i, j}^{k-1} .
\end{aligned}
$$

The constants $A_{i}(i=1, \ldots, 4)$ in $\Omega_{1}$ and $\Omega_{2}$ are different. Let $A_{i}^{-}$and $A_{i}^{+}$ be the $A_{i}$ values in $\Omega_{1}$ and $\Omega_{2}$, respectively. To take into account the continuity conditions at the line $x_{1}=a, x_{2} \in(-b, b)$ we choose the coefficients $b_{i j}$ as follows:

$$
\begin{aligned}
& b_{11}=b_{12}=A_{1}^{-} \frac{\tau^{2}}{h_{1}^{2}}, \quad b_{13}=A_{2}^{-} \frac{\tau^{2}}{h_{2}^{2}}, \quad b_{14}=b_{15}=A_{3}^{-} \frac{\tau^{2}}{4 h_{1} h_{2}}, \\
& b_{21}=b_{22}=A_{2}^{-} \frac{\tau^{2}}{h_{1}^{2}}, \quad b_{23}=A_{4}^{-} \frac{\tau^{2}}{h_{1}^{2}}, \quad \text { if } i=1,2, \ldots, N_{1}^{t}, \\
& b_{11}=\frac{A_{1}^{+}+A_{1}^{+}}{2} \frac{\tau^{2}}{h_{1}^{2}}, \quad b_{12}=A_{1}^{-} \frac{\tau^{2}}{h_{1}^{2}}, \quad b_{13}=A_{2}^{-} \frac{\tau^{2}}{h_{2}^{2}}, \quad b_{14}=\frac{A_{3}^{-}+A_{3}^{+}}{2} \frac{\tau^{2}}{4 h_{1} h_{2}},
\end{aligned}
$$


$b_{15}=A_{3}^{-} \frac{\tau^{2}}{4 h_{1} h_{2}}, \quad b_{21}=\frac{A_{2}^{-}+A_{2}^{+}}{2} \frac{\tau^{2}}{h_{1}^{2}}, \quad b_{22}=A_{4}^{-} \frac{\tau^{2}}{h_{2}^{2}}, \quad b_{23}=A_{4}^{-} \frac{\tau^{2}}{h_{2}^{2}}, \quad$ if $i=N_{1}^{t}$,

$b_{11}=A_{1}^{+} \frac{\tau^{2}}{h_{1}^{2}}, \quad b_{12}=\frac{A_{1}^{-}+A_{1}^{+}}{2} \frac{\tau^{2}}{h_{1}^{2}}, \quad b_{13}=A_{2}^{+} \frac{\tau^{2}}{h_{2}^{2}}, \quad b_{14}=A_{3}^{+} \frac{\tau^{2}}{4 h_{1} h_{2}}$,

$b_{15}=\frac{A_{3}^{-} \tau^{2}}{4 h_{1} h_{2}}, \quad b_{21}=A_{2}^{+} \frac{\tau^{2}}{h_{1}^{2}}, \quad b_{22}=\frac{A_{2}^{-}+A_{2}^{+}}{2} \frac{\tau^{2}}{h_{1}^{2}}, \quad b_{23}=A_{4}^{+} \frac{\tau^{2}}{h_{2}^{2}}$ if $i=N_{1}^{t}+1$,

$b_{11}=b_{12}=A_{1}^{+} \frac{\tau^{2}}{h_{1}^{2}}, \quad b_{13}=A_{2}^{+} \frac{\tau^{2}}{h_{1}^{2}}, \quad b_{14}=b_{15}=A_{3}^{+} \frac{\tau^{2}}{4 h_{1} h_{2}}$,

$b_{21}=b_{22}=A_{2}^{+} \frac{\tau^{2}}{h_{1}^{2}}, \quad b_{23}=A_{4}^{+} \frac{\tau^{2}}{h_{2}^{2}}, \quad$ if $i=N_{1}^{t}+2, \ldots, N_{1}-1$.

Discretizing the boundary conditions we get

$$
\begin{aligned}
& A_{1111} \frac{\bar{u}_{1 j}^{k}-\bar{u}_{0 j}^{k}}{h_{1}}+A_{2211} \frac{\overline{\bar{u}}_{0, j+1}^{k}-\overline{\bar{u}}_{0, j-1}^{k}}{2 h_{2}}=\mu_{j}^{k} \cos \alpha, \\
& A_{1212} \frac{\overline{\bar{u}}_{0, j+1}^{k}-\overline{\bar{u}}_{0, j-1}^{k}}{2 h_{2}}+\frac{\bar{u}_{1, j}^{k}-\bar{u}_{0, j}^{k}}{h_{1}}=\mu_{j}^{k} \sin \alpha
\end{aligned}
$$

where

$$
\begin{aligned}
& \mu_{j}^{k}= \begin{cases}B\left(t_{k}, x_{2 j}\right), & \text { if } t_{k} \leq 1, \text { and }-b \varepsilon \leq x_{2} \leq b \varepsilon \\
0 & \text { otherwise; }\end{cases} \\
& A_{1111} \frac{\bar{u}_{N_{1}, j}-\bar{u}_{N_{1}-1, j}}{h_{1}}+A_{2211} \frac{\overline{\bar{u}}_{N_{1}, j+1}-\overline{\bar{u}}_{N_{1}, j-1}}{2 h_{2}}=0 \\
& \frac{\bar{u}_{N_{1}, j+1}-\bar{u}_{N_{1}, j-1}}{2 h_{2}}+\frac{\overline{\bar{u}}_{N_{1}, j}-\overline{\bar{u}}_{N_{1}-1, j-1}}{h_{1}}=0, \text { if } j=1,2, \ldots, N_{2}-1, \\
& A_{2211} \frac{\bar{u}_{i+1,0}-\bar{u}_{i-1,0}}{2 h_{1}}+A_{2222} \frac{\overline{\bar{u}}_{i, 1}-\overline{\bar{u}}_{i, 0}}{h_{2}}=0, \\
& \frac{\bar{u}_{i, 1}-\bar{u}_{i, 0}}{h_{2}}+\frac{\overline{\bar{u}}_{i+1,0}-\overline{\bar{u}}_{i-1,0}}{2 h_{1}}=0, \\
& A_{2211} \frac{\bar{u}_{i+1, N_{2}}-\bar{u}_{i-1, N_{2}}}{2 h_{1}}+A_{2222} \frac{\overline{\bar{u}}_{i, N_{2}}-\overline{\bar{u}}_{i, N_{2}-1}}{h_{2}}=0 \\
& \frac{\bar{u}_{i, N_{2}}-\bar{u}_{i, N_{2}-1}}{h_{2}}+\frac{\overline{\bar{u}}_{i+1, N_{2}}-\overline{\bar{u}}_{i-1, N_{2}}}{2 h_{1}}=0, \quad \text { if } i=1,2, \ldots, N_{1}-1 .
\end{aligned}
$$

To determine

$$
\bar{u}_{0,0}^{k+1}, \quad \overline{\bar{u}}_{0,0}^{k+1}, \quad \bar{u}_{0, N_{2}}^{k+1}, \quad \overline{\bar{u}}_{0, N_{2}}^{k+1}, \quad \bar{u}_{N_{1}, 0}^{k+1}, \quad \overline{\bar{u}}_{N_{1}, 0}^{k+1}, \quad \bar{u}_{N_{1}, N_{2}}^{k+1}, \quad \overline{\bar{u}}_{N_{1}, N_{2}}^{k+1}
$$


Numerical Analysis of the Wave Propagation in a Composite Medium 169

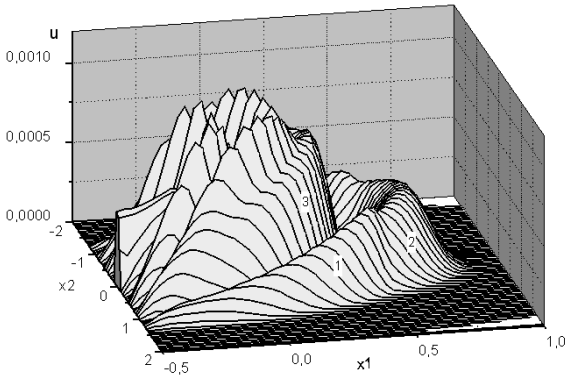

(a)

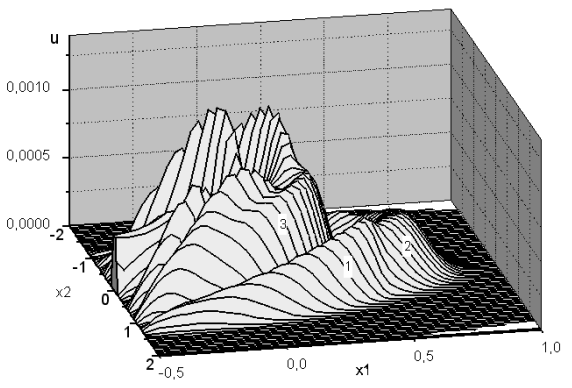

(b)

Figure 6. The graph of $|u| . \xi_{1}=0.2, \xi_{2}=0.4, t=2$. (a) $\alpha=0$; (b) $\alpha=\pi / 6$.

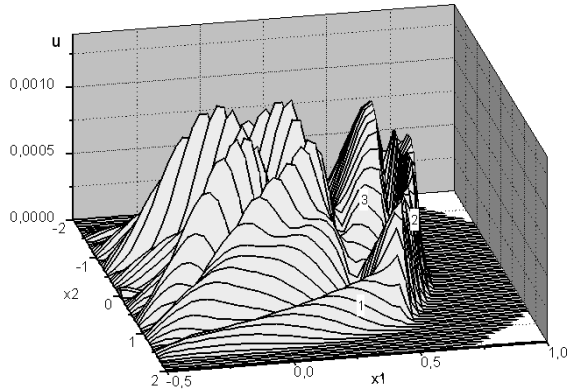

(a)

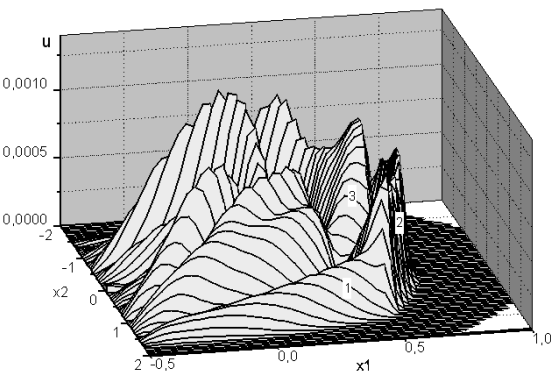

(b)

Figure 7. The graph of $|u| . \xi_{1}=0.3, \xi_{2}=0.032, t=2$. (a) $\alpha=0$; (b) $\alpha=\pi / 6$.

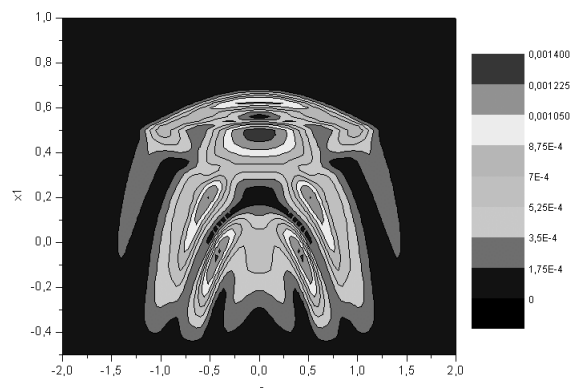

(a)

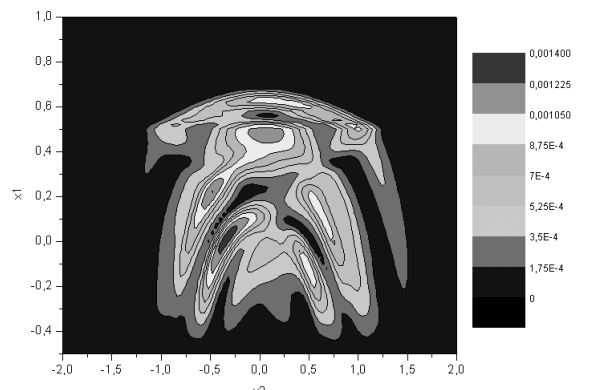

(b)

Figure 8. Isolines of $|u| . \xi_{1}=0.3, \xi_{2}=0.032, t=2$. (a) $\alpha=0$; (b) $\alpha=\pi / 6$.

we use values from $k$ th and $(k+1)$ th time layers that are already determined:

$\bar{u}_{0,0}^{k+1}=\frac{\bar{u}_{0,0}^{k}+\bar{u}_{0,1}^{k}+\bar{u}_{1,0}^{k}+\bar{u}_{1,1}^{k+1}}{4}, \quad \overline{\bar{u}}_{0, N_{2}}^{k+1}=\frac{\overline{\bar{u}}_{0, N_{2}}^{k}+\overline{\bar{u}}_{0, N_{2}-1}^{k}+\overline{\bar{u}}_{1, N_{2}}^{k}+\overline{\bar{u}}_{1, N_{2}-1}^{k+1}}{4}$.

The discretized initial conditions are the following 


$$
\begin{aligned}
& \bar{u}_{i j}^{0}=0, \quad \overline{\bar{u}}_{i j}^{0}=0, \quad \bar{u}_{i j}^{1}=\bar{u}_{i j}^{0}, \quad \overline{\bar{u}}_{i j}^{1}=\overline{\bar{u}}_{i j}^{0}, \\
& i=0,1, \ldots, N_{1}, j=0,1, \ldots, N_{2} .
\end{aligned}
$$

This allows us to solve four systems of linear equations (4.1), (4.2), (4.3) and (4.4) separately and so essentially reduce CPU time. The matrix of each such system consists of four differently located blocks. Two blocks are diagonal matrixes and the other two ones are of two diagonal type.

The local approximation error for inner points of $\Omega_{1} \cup \Omega_{2}$ is $O\left(\tau^{2}+h_{1}^{2}+h_{2}^{2}\right)$. The discrete boundary conditions are given with the accuracy $O\left(h_{1}+h_{2}^{2}\right)$ at the lines $x_{1}=-a$ and $x_{1}=d$ and $O\left(h_{1}^{2}+h_{2}\right)$ at $x_{2}= \pm b$, respectively. At the line $x_{1}=a$ we have the local approximation of the second order with respect to $t$ and $x_{2}$. The difference sheme is stable if the grid parameters $h_{1}, h_{2}$, and $\tau$ satisfy the inequality

$$
\tau=\frac{\min \left(h_{1}, h_{2}\right)}{\sqrt{4 \max _{i=1, \ldots, 4}\left(A_{i}^{-}, A_{i}^{+}\right)}} .
$$

We performed several experiments with different values of $\tau, h_{1}$, and $h_{2}$. The results presented in this section are computed with

$$
a=0.5, d=1, b=2, h_{1}=0.01, h_{1}=0.02, \tau=0.005 .
$$

The numerical experiments for different values of $\tau, h_{1}$, and $h_{2}$ show that the numerical solution converges to the solution of problem (3.1) and (3.2).

In the 2-dimensional case we used the following constants:

$$
\begin{aligned}
& T=10^{-5} \mathrm{~s}, \quad L=200 \mathrm{~mm}, \quad \rho=7.9 \cdot 10^{-3} \mathrm{~g} / \mathrm{mm}^{3}, \eta=1000, \varepsilon=0.1, \\
& \tilde{E}_{1}=2.3, \quad \tilde{E}_{2}=1.9, \quad \tilde{G}_{12}=0.8, \quad \xi_{1}=0.3, \quad \xi_{2}=0.032
\end{aligned}
$$

and $\xi_{1}=0.2, \xi_{2}=0.4$. Numerical results of the waves diffraction problem are presented in Figs. 6-9. In Fig. 6(a) the graph of $|u|$ is presented for $\xi_{1}=$ $0.2, \xi_{2}=0.4, \alpha=0$, and $t=2$. Fig. 6 (b) represents the graph of $|u|$ for $\xi_{1}=0.2, \xi_{2}=0.4, \alpha=\pi / 6$, and $t=2$. In both $6(\mathrm{a})$ and $6(\mathrm{~b})$ figures waves 1 and 2 illustrate longitudinal waves $(\operatorname{div} u \neq 0)$ while waves 3 are transversal (div $u=0$ ). In Figs. 7(a) and 7(b) the graph of $|u|$ is represented for $t=2$, $\xi_{1}=0.3, \xi_{2}=0.032, \alpha=0$, and $\alpha=\pi / 6$, respectively. Here waves 1 and 2 are longitudinal and waves 3 are transversal. Figures 6 and 7 show, that support of wave in domain $\Omega_{2}$ decreases with $\xi_{2} / \xi_{1}$ decreasing. In Figs. 8(a) and $8(\mathrm{~b})$ the isolines of $|u|$ are represented for data given in Figs. 7(a) and 7 (b).

Figures 9(a)-(f) represent the dynamics of the waves interaction with the free boundary $\left(x_{1}=d=a\right)$ for $\alpha=0, \xi=0.032$, and $t=4,4.25,4.5,4.75,5$ and 5.25, respectively. In Figs. 9(d)-(f) we can see also the interaction of waves moving forward and back. 


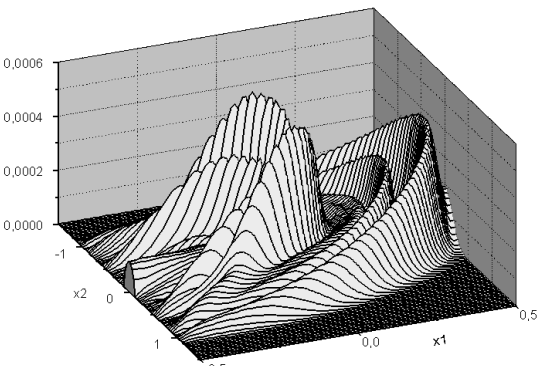

(a)

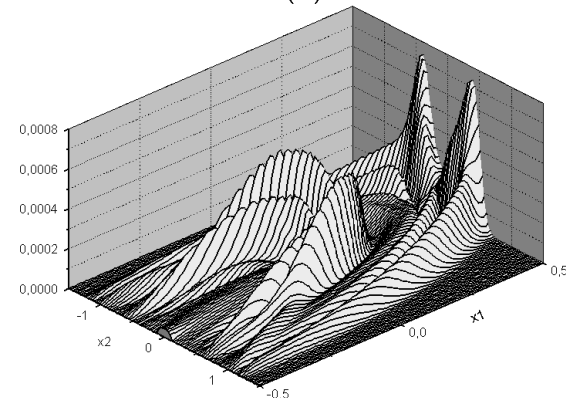

(c)

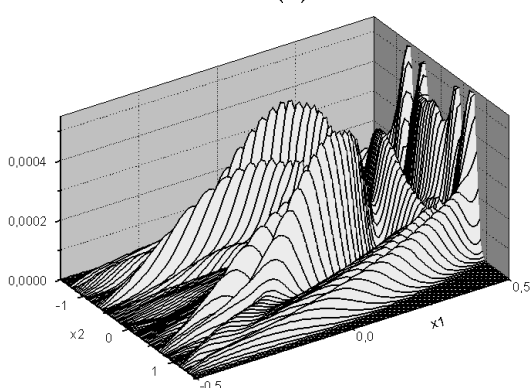

(e)

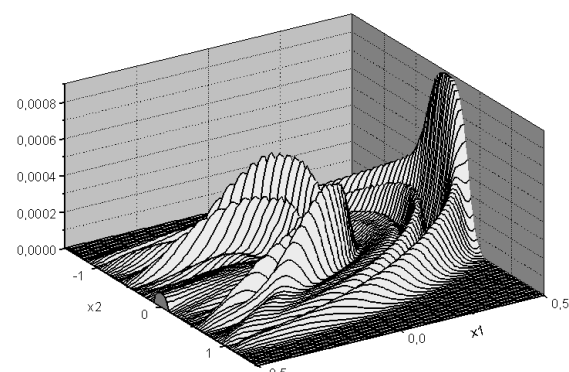

(b)

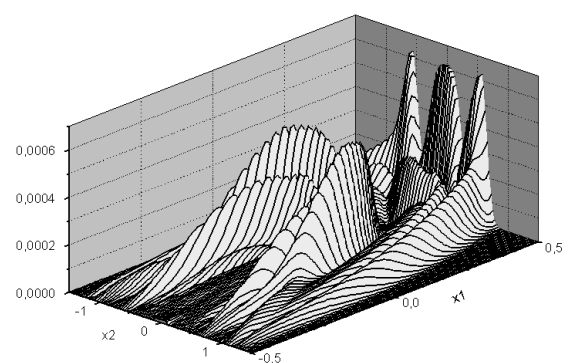

(d)

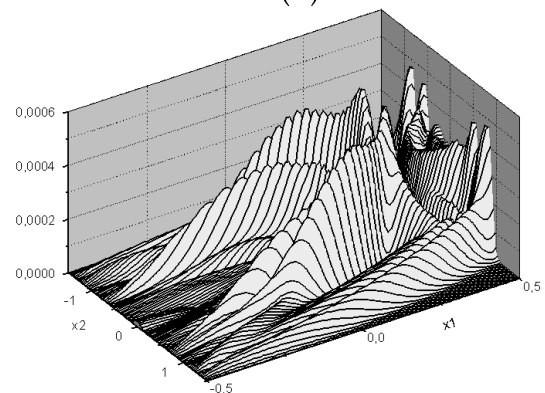

(f)

Figure 9. The graph of $|u| . \xi=0.032$, (a) $t=4$; (b) $t=4.25$; (c) $t=4.5$; (d) $t=4.75$; (e) $t=5$; (f) $t=5.25$.

\section{Concluding Remarks}

1. The diffraction and waves interaction with a free boundary problems are examined numerically and analytically in a material structure composed of random oriented orthotropic crystals. In the two-dimensional case only the numerical solution is examined.

2. The dynamics of the longitudinal and transversal waves for both problems are studied.

3. The waves increase (decrease) in the domains $\Omega_{2}$ and $(1, d)$ with $\xi_{2}<\xi_{1}$ and $b_{2}<b_{1}\left(\xi_{2}>\xi_{1}\right.$ and $\left.b_{2}>b_{1}\right)$, respectively, is obtained. Because of the interference the waves increase at the free boundary in one-dimensional problem is observed. 
4. In two-dimensional problem the waves interaction with the free boundary is more complicated. Since waves are not plane, then different parts of these waves reach the free boundary at the different moments. Some parts of a wave move forward while the other ones move back. The interaction of reflected waves and the other ones moving forward complicates the analysis (see Figs. 9(d)-(f)).

5. In the symmetric case the wave dynamics on the line of symmetry is similar to that of the one-dimensional problem.

\section{References}

[1] Buryachenko, V.A., N.J. Pegano, R.J. Kim and J.E. Spowart. Quantitative description and numerical simulation of random microstructure of composites and their effective elastic moduli. Int. J. Solids and Structures, 40, 47-72, 2003.

[2] J. Dabulytè, F. Ivanauskas, V. Skakauskas and R. Barauskas. The structure modelling of material composed of the orthotropic crystals. Nonlinear Analysis (Modelling and Control), 9(4), 297-306, 2004.

[3] S.H. Ju and Y.M. Wang. Time-dependent absorbing boundary conditions for elastic wave propagation. Int. J. Math. Eng., 50, 2159-2174, 2001.

[4] A.A. Samarskii. The Theory of Difference Schemes. Nauka, Moscow. 1989 (In Russian) 